\title{
The Effect of Well Site and interlayer permeability on Recovery Factor of Horizontal Well-High Concentration Polymer Flooding in Lateral Accretion Layer
}

\author{
Taoping Chen ${ }^{1, a}$, Qiang $\mathrm{Li}^{1, \mathrm{~b}}$ \\ ${ }^{1}$ The Key Laboratory of Enhancing Oil and Gas Recovery Efficiency of Educational Ministry, \\ Northeast Petroleum University, Daqing 163318, Heilongjiang, China. \\ actp010@163.com, bli361qiang@163.com
}

\begin{abstract}
Keywords: Lateral accretion layer, Recovery factor, Horizontal well, Well site, Interlayer permeability

Abstract. CMG software was used to build an ideal model whose size ratio is 1:1:0.1, there are three completely closed interlayers whose dip angle is $10^{\circ}$ in the upper model, and the lower model is connected horizontal layer. The thickness ratio between the upper model with the lower model is 2:1, and the permeability ratio is $1: 1.8$.The water well and the oil well are arranged at the central position of the model's ends along the extending direction of lateral accretion layer, and the horizontal well is arranged at the top of lateral accretion layer on the line of water well to oil well. The angles of the main streamline to the extending direction of lateral accretion layer, the interlayer permeability and the deviation position of the horizontal well in two injection-two production mode are researched on the basis of conventional water flooding-polymer flooding and horizontal well-high concentration polymer flooding. The results show that, as the angle of the main streamline to the extending direction of lateral accretion layer increases, the overall recovery factor decreases. The factor decreases by $5.96 \%$ from the angle of $0^{\circ}$ to $45^{\circ}$. As the horizontal well deviates to one side, the overall recovery factor is reduced. The factor decreases by $4.66 \%$ from the center position to the maximum deviation position. Finally with the reduction of the interlayer permeability, the recovery factor of the horizontal well-high concentration polymer flooding increases. The factor can reach $14 \%$ when the interlayer is completely closed, while the factor is $4.9 \% \sim 4.5 \%$ when the permeability ratio between interlayer with lateral accretion layer is $0.2 \sim 0.3$.
\end{abstract}

\section{Introduction}

Lateral accretion layer in fluvial reservoir, is divided into two kinds of interlayer of sedimentation and diagenesis from the origin. While lateral accretion layer plays a role in blocking injected water, leading to a large number of remaining oil in the lateral accretion layer at the top of the reservoir which can not be displaced after the straight well water flooding. The potential can be dug by taking on the method of digging the horizontal well at the top of the reservoir combing with chemical flooding. Jian Wang, Xiaoxiang Qi, Lei Wang, et al analyzed and described the formation and distribution of the lateral accretion layer in fluvial reservoir ${ }^{[1-3]}$. Shanyan Zhang, Dali Yue, Hongen Dou et al predicted and described the lateral accretion layer preliminarily ${ }^{[4-7]}$. Dandan hu, Qinglin Shu, et al studied the influence of lateral accretion layer on the remaining oil distribution ${ }^{[8-9]}$. Changming Liu, Xiuli Liang, Wenfu Cui et al learned the influence degree of the dip angle, horizontal spacing and other factors of the lateral accretion layer on the overall recovery factor, by using numerical simulation software to establish a conceptual model ${ }^{[10-14]}$. Chao Jiang analyzed the influence of different water flooding direction, quantity of injection and interlayer condition on the overall recovery factor and remaining oil distribution , and optimized the position of horizontal well and injection-production well types, by using one injection-one production conceptual mode ${ }^{[15]}$. There is few discussion about the well site and the interlayer permeability for now. In this paper, CMG software is used to calculate the influence of the angles of the main streamline to the extending direction of lateral accretion layer, the interlayer permeability and the deviation position of the 
horizontal well in two injection-two production mode on recovery factor, and further research the effect of well site and interlayer permeability on recovery factor of horizontal well-high concentration polymer flooding in lateral accretion.

\section{Building conceptual model}

Build a conceptual model which grid number is $36 * 17 * 18$ and mesh size is $5.01 \mathrm{~m} * 10.609 \mathrm{~m} * 0.879 \mathrm{~m}$ by CMG simulation software. And model is divided into 18 floors vertically, with 11016 grids. There are three interlayers whose dip angle is $10 \mathrm{o}$ in the upper model, and the lower model is connected horizontal layer. Interlayers are completely closed in the conceptual model and the permeability is $0 \mu \mathrm{m}^{2}$. While the upper model permeability is $1000 \mu \mathrm{m}^{2}$, and the lower model permeability is $1800 \mu \mathrm{m}^{2}$.

Inject well W-1 and produce well W-2 are arranged in water flooding and polymer flooding period. $\mathrm{W}-2$ is shut after polymer flooding, andW-2iw is arranged and at the original position, while arranging the horizontal well $\mathrm{W}-3$ as shown in Figure 1.

Other basic data of the model as follows, the porosity is 0.25 , net to gross is 1 , top depth is $1000 \mathrm{~m}$, original oil saturation is 0.733 , water saturation is 0.267 , viscosity of the oil is $9 \mathrm{mPa}$.s and viscosity of the water is $0.6 \mathrm{mPa}$.s.

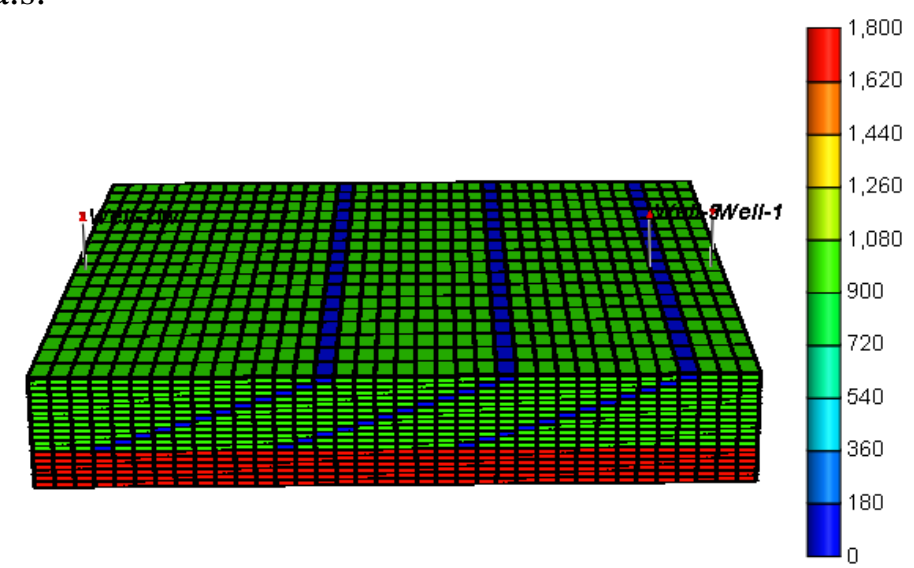

Fig.1 The schematic diagram of the ideal model

\section{Numerical simulation results and analysis}

Horizontal well-high concentration polymer flooding scheme and recovery factor of the conceptual model. Water flooding: vertical wells W-1 inject and W-2 produce, constant injection $80 \mathrm{~m}^{3} /$ day, water flooding until the water content can be up to $98 \%$.

Polymer flooding: vertical wells W-1 inject and W-2 produce, constant injection $80 \mathrm{~m}^{3} / \mathrm{day}$, subsequent water flooding after polymer flooding $0.3 \mathrm{PV}(1600 \mathrm{mg} / \mathrm{L})$ until the water content can be up to $98 \%$.

Horizontal well-high concentration polymer flooding: shut the W-2, open the W-2iw with W-1, constant injection $40 \mathrm{~m}^{3}$ / day, horizontal wells W-3 produce, subsequent water flooding after polymer flooding 0.3PV $(1900 \mathrm{mg} / \mathrm{L})$ until the water content can be up to $98 \%$.

Draw the oil recovery factor figure and water content figure with the numerical simulation results as shown in Figure 2.

The recovery factor of water flooding is $21.87 \%$, this part of oil is mainly existed in the high permeability layer. Because there are interlayers in the upper model, the injected water mainly get to the lower model. And the high permeability layer has large pore and small resistance, which can be conducive to flow. The increase of the recovery factor of polymer flooding is $5.994 \%$. Polymer solution can increase the viscosity of injected water, reduce the permeability of water phase and expand the water sweep volume. Plug high permeable layer on the vertical to get the displacement of residual oil in low permeability layer. The recovery factor of horizontal well-high concentration 
polymer flooding is $13.96 \%$. Remaining oil in the upper model which can not be displaced by vertical after is displaced partly after opening the upper horizontal well. We can determine that the horizontal well-high concentration polymer flooding can effectively improve the recovery factor after polymer flooding.

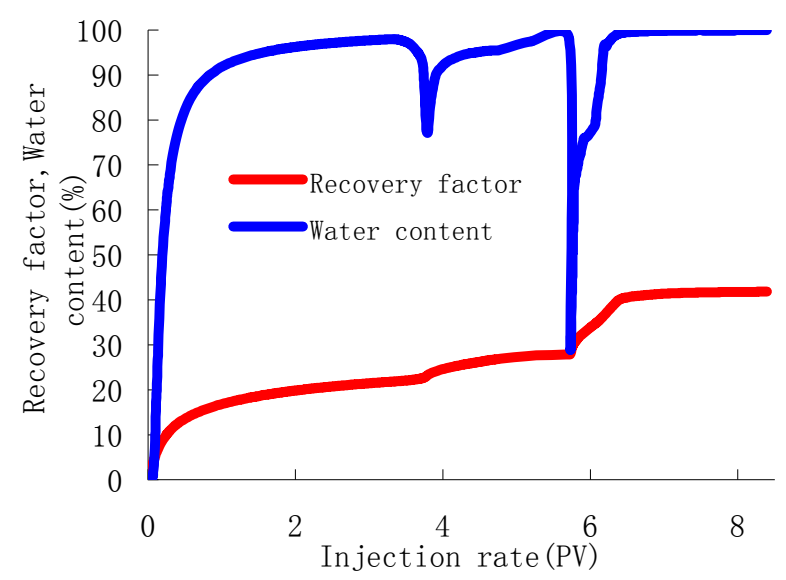

Fig 2. The oil recovery factor and water content of streamline

foundation scheme

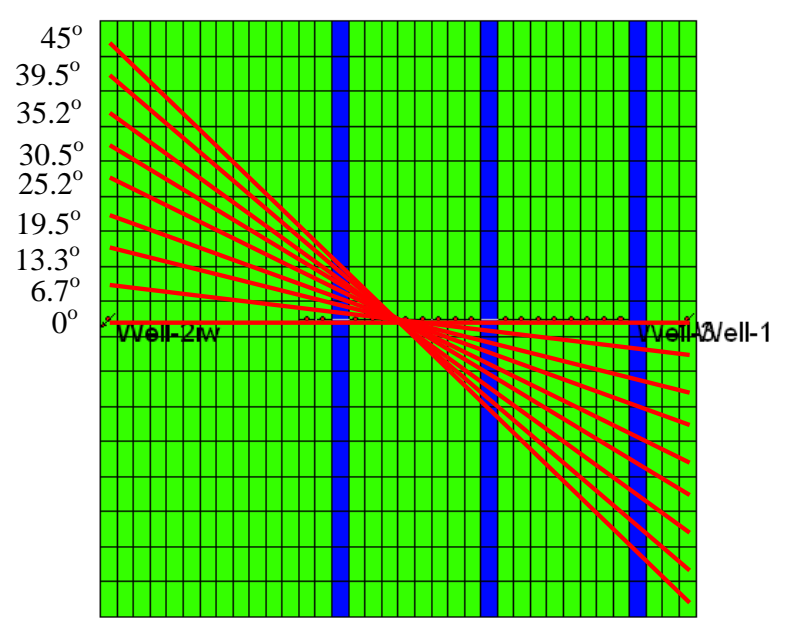

Fig.3 Variation range of the angles of the main

(t) accretion layer on recovery factor. In the conceptual model, the main streamline is parallel with the extending direction of lateral accretion layer. Now by changing the well site of $\mathrm{W}-1$ and $\mathrm{W}-2$, change the angle of the main streamline to the extending direction of lateral accretion layer to $0^{\circ}, 6.7^{\circ}, 13.3^{\circ}$, $19.5^{\circ}, 25.2^{\circ}, 30.5^{\circ}, 35.2^{\circ}, 39.5^{\circ}, 45^{\circ}$ as shown in Figure 3.

Draw the relationship of the oil recovery factor with the injection rate at different angles of the main stream line to the extending direction of lateral accretion layer as shown in Figure 4, and the relationship of each stage oil recovery factor with the angles of the mainstream line to the extending direction of lateral accretion layer as shown in Figure 5.

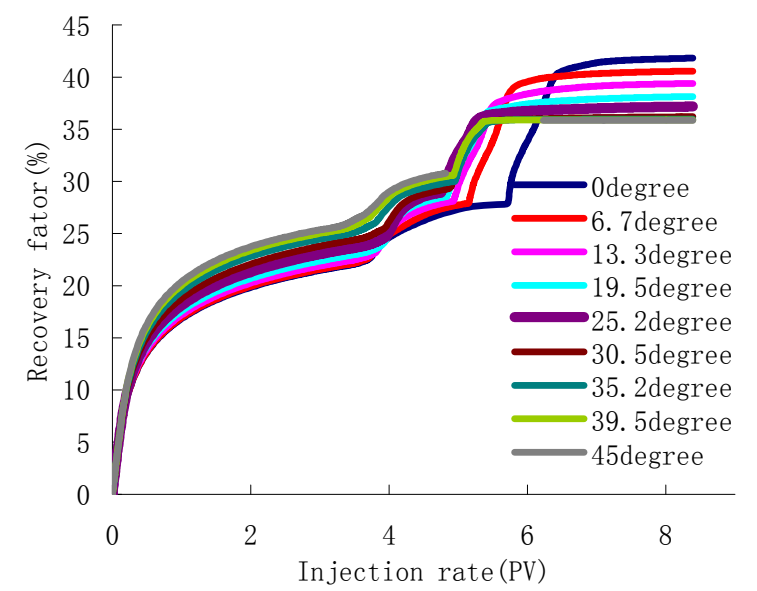

Fig.4 The relationship of the oil recovery factor with the injection rate at different angles of the mainstream line to the extending direction of lateral accretion layer

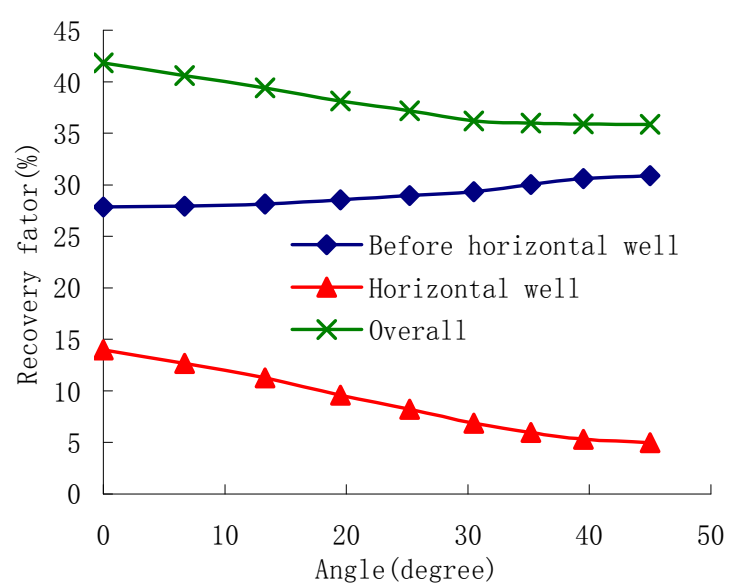

Fig. 5 The influence of the angles of the mainstream line to the extending direction of lateral accretion layer on each stage oil recovery factor

By Figure 5 we can see, as the angle of the mainstream line to the extending direction of lateral accretion layer increases, water flooding-polymer flooding recovery factor increases, horizontal well-high concentration polymer flooding recovery factor decreases and the overall recovery factor decreases. Analysis the reason for this is that, in the water flooding-polymer flooding stage, as the angle of the mainstream line to the extending direction of lateral accretion layer increases, it makes the mainstream line distance and sweeping area increases. Thus improving the use degree of the remaining oil, recovery factor increases. But in the stage of horizontal well-high concentration polymer flooding, as the angle of the mainstream line to the extending direction of lateral accretion 
layer increases, sweeping degree to the lateral accretion layer recede. The recovery factor of horizontal well-high concentration polymer flooding reduces obviously, leading to the overall recovery factor lower.

The influence of interlayer permeability on recovery factor. In the conceptual model, the permeability of lateral accretion layer is set to $0 \mu \mathrm{m}^{2}$, but the actual layer is not completely closed, so now we modify the interlayer permeability as $1 \%, 5 \%, 10 \%, 20 \%, 30 \%, 50 \%, 80 \%, 100 \%$ of the lateral accretion layer permeability.

Draw the relationship of the oil recovery factor with the injection rate at different interlayer permeablity as shown in Figure 6, and the relationship of each stage oil recovery factor with the ratio of the interlayer permeability with the lateral accretion layer permeability as shown in Figure 7.

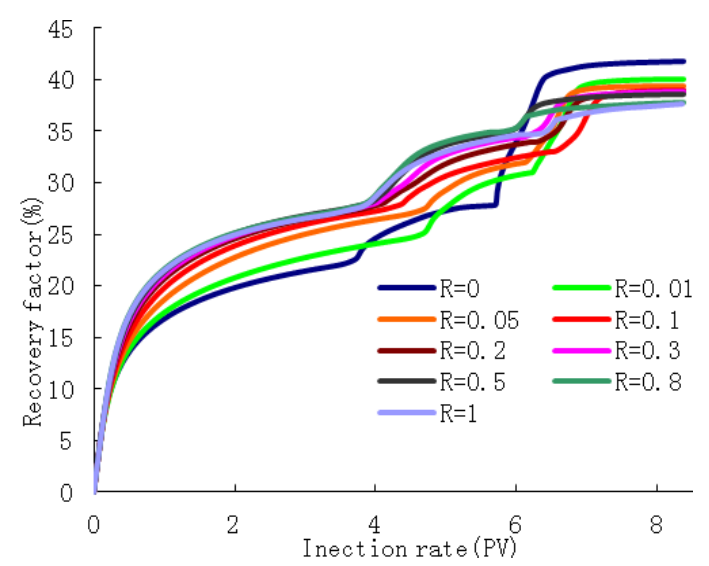

Fig.6 The relationship of the oil recovery factor with the injection rate at different permeability ratio

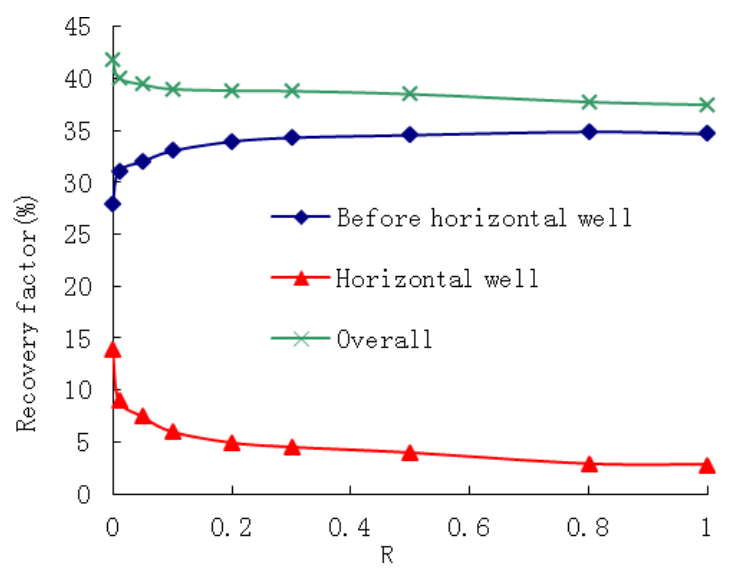

Fig.7 The influence of the permeability ratio on each stage of oil recovery factor

By Figure 7 we can see, as the interlayer permeability increases, water flooding-polymer flooding recovery factor increases, horizontal well-high concentration polymer flooding recovery factor decreases and the overall recovery factor decreases. Analysis of the reason is that, with the interlayer permeability becoming higher, leading to more used of remaining oil in the lateral accretion layer, water flooding-polymer flooding recovery factor increases. It is the earlier used of remaining oil in the lateral accretion layer what leads to seriously impact on horizontal well-high concentration polymer flooding recovery factor and the reduce of the overall recovery factor.

The influence of deviation position of the horizontal well in two injection-two production mode on recovery factor. There is a inject well $\mathrm{W}-1$ and a produce Well $\mathrm{W}-2$ in the ideal model, but there are may two groups of inject-produce wells in element pattern. Now set a new group of inject-produce wells W-5 and W-6, and arrange the horizontal well between the two groups of inject-produce wells, as shown in Figure 8. Shutin W-2 and W-6 after polymer flooding, open W-2i and W-6i and start horizontal well-high concentration polymer flooding. Compare the recovery factor of two injection-two production mode and one injection- one production mode after getting the data. Then deviate the horizontal well to study the influence of deviation position of the horizontal well in two injection-two production mode on recovery factor. The original location for horizontal well is "Deviate 0" in Figure 8, the biggest deviation position is "Deviate1" in Figure 8. Research the influence on the recovery factor when deviation position of horizontal well is $0,1 / 8,1 / 4,3 / 8,1 / 2,5 / 8$, 3/4, 7/8, 1 .

Draw the relationship of the oil recovery factor with the injection rate at different deviation positions of the horizontal well as shown in Figure 9, and the relationship of each stage oil recovery factor with the deviation positions of the horizontal well as shown in Figure 10.

By Figure 9 we can see that the recovery factors of every stages of two injection-two production mode are higher than one injection-one production mode. By Figure 10 we can see that as the horizontal well deviates, horizontal well-high concentration polymer flooding recovery factor decreases and the overall recovery factor decreases. Analysis of the reason is that, as horizontal well shifts to one side, the function of the other side injection decreases, leading to lower horizontal 
well-high concentration polymer flooding recovery factor. Therefore the overall recovery facotor decreases based on the same water flooding-polymer flooding recovery factor.

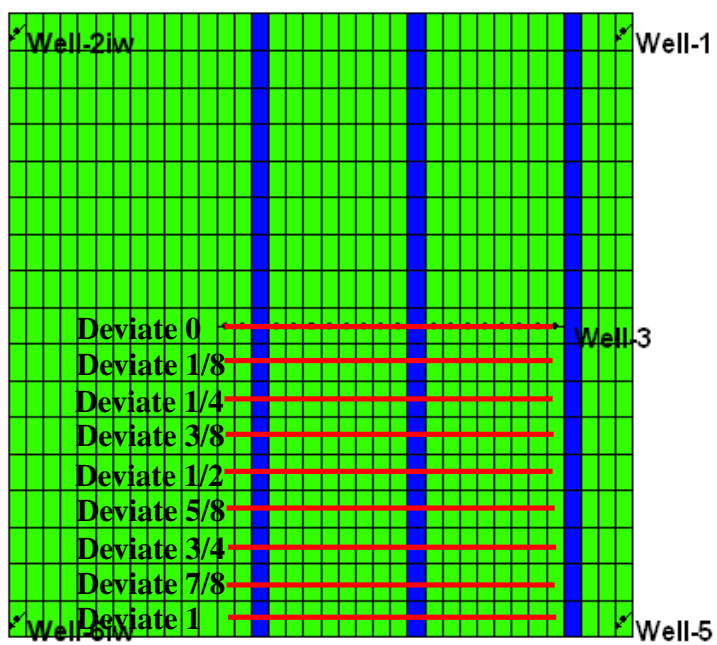

Fig.8 Deviation position of the horizontal well

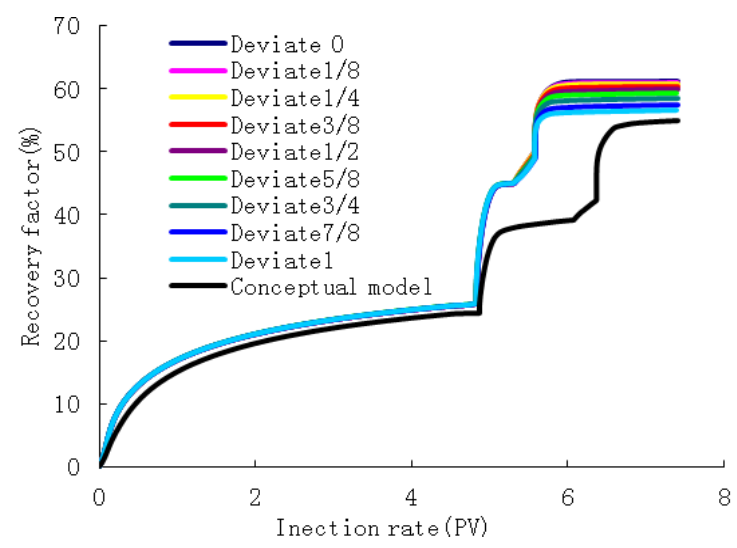

Fig.9 The relationship of the oil recovery factor with the injection rate at different deviation position of the horizontal well

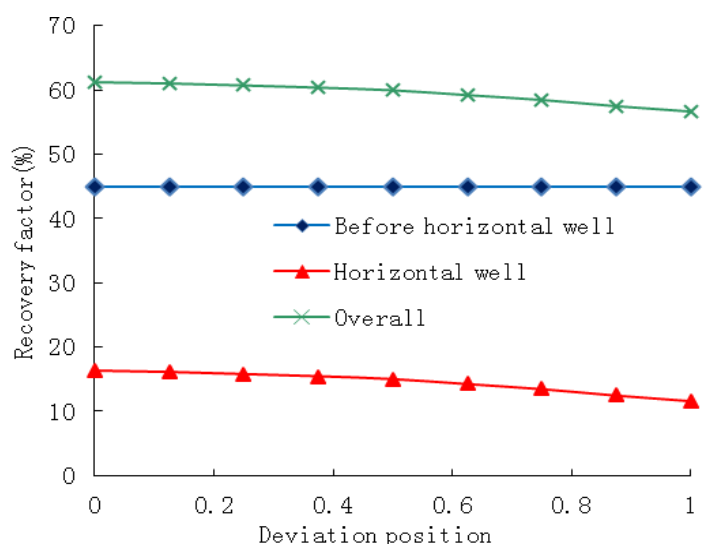

Fig.10 The influence of the deviation position of the horizontal well on each stage of oil recovery factor of two inject-two produce mode

\section{Conclusion}

1) As the angle of the mainstream line to the extending direction of lateral accretion layer decreases, horizontal well-high concentration polymer flooding recovery factor increases and the overall recovery factor increases. The horizontal well-high concentration polymer flooding recovery factor increases by $8.97 \%$ and the overall factor increases by $5.96 \%$ from the angle of $45^{\circ}$ to $0^{\circ}$. So arranging injection- production well parallel to the extending direction of lateral accretion layer can improve oil recovery factor in allusion to lateral accretion layer chemical flooding on horizontal well.

2) With the reduction of the interlayer permeability, the recovery factor of the horizontal well-high concentration polymer flooding increases and the overall recovery factor increases. The factor can reach $14 \%$ when the interlayer is completely closed, while the factor is $4.9 \% \sim 4.5 \%$ when the permeability ratio between interlayer with lateral accretion layer is 0.2 0.3.

3) The recovery factors of every stages of two injection-two production mode are higher than one injection-one production mode. As the horizontal well deviates to one side, the overall recovery factor is reduced. The factor decreases by $4.66 \%$ from the center position to the maximum deviation position

\section{Acknowledgements}

The project was supported by National Science and Technology Major Project of the Ministry of Science and Technology of China (2011ZX05009-004-002). 


\section{References}

[1] Jian Wang, Shouyu Xu, Weiping Zhong. Fluvial facies reservoir interlayer formation and its distribution characteristics[J]. Geological Science and Technology Information, 2010, 19(4):84-88. (In Chinese)

[2] Xiaoxiang Qi, Deyong Ma, Lei Liu, Wei Li, Shaoming Song. The channel sand body internal intercalation characteristics research[J]. Daqing petroleum geology and development, 2005, 24(3):14-16. (In Chinese)

[3] Lei Wang, Guotao Liu, Tao Long, Chi Ma. A description method of lateral accretion layer in point bar of meandering river[J]. Lithologic oil-gas reservoir, 2008, 14(2):23-26. (In Chinese)

[4] Shanyan Zhang, Bo Liu, Guofei Chen, et al. Horizontal well core side interlining[J]. Daqing petroleum geology and development, 2007, 26(6):58-59. (In Chinese)

[5] Hongen Dou, Changzhi Guan, Shengjiang Lian. The experimental studies of physical simulation of bottom water reservoirs with barrier and permeable interbed on horizontal well[J]. SPE, 2008.

[6] Allen JRL. Studies in fluviatile sedimentation: bars, bar complexes and sandstone sheets (lower-sinuosity braided streams) in the Brownstones(L.Devonian), Welsh Bord ers. Sediment Geol. 2002, 33:237-293.

[7] Dali Yue. Meandering river reservoir conformation analysis and residual oil distribution pattern research in Gudao oilfield as an example[D].China Petroleum University, 2006. (In Chinese)

[8] Dandan Hu, Wei Tang, Yuwen Chang, et al. The research on the influence of residual oil from interlayer in the thick oil layers. Special oil and gas reservoirs, 2009, 16(3):50-51. (In Chinese)

[9] Qinglin Shu. The channel sand side body has influence on remaining oil distribution-To an island oilfield pavilion in 3 4 high sinuosity of sand group on the meandering river as an example[J]. Oil-gas geology and recovery, 2005, 12(2):45-46. (In Chinese)

[10] Changming Liu. The remaining oil distribution after polymer flooding and extract technology research of Pu-1 Group[D]. Northeast Petroleum University, 2010. (In Chinese)

[11] Xiuli Liang. Comprehensive description of remaining oil distribution after polymer flooding of Pu-1 Group[D]. Northeast Petroleum University, 2010. (In Chinese)

[12] Wenfu Cui. Fluvial facies reservoir layer upon layer structure and residual oil distribution study of Gudong Oilfield-7- West pavilion[D]. China Petroleum University(Beijing),2008.(In Chinese)

[13] Wei Zhou, Zhonghua Tang, Jing Wen, et al. Application of numerical simulation technology on researching of remaining oil distribution law [J]. Fault-Block Oil \& Gas Field, 2010, 17(3):327-328. (In Chinese)

[14] Lei Wang, Ziqiang Tao. Numerical simulation study Inside lateral accretion layer in the meandering river point bar[J]. Complex Hydrocarbon Reservoirs, 2011, 24(3):14-16. (In Chinese)

[15] Chao Jiang. Numerical simulation of remaining oil of lateral accretion layer after horizontal well polymer flooding[D]. Northeast Petroleum University, 2013. (In Chinese) 